\title{
APOL1 variants change C-terminal conformational dynamics and binding to SNARE protein VAMP8
}

Sethu M. Madhavan, ${ }^{1}$ John F. O'Toole, ${ }^{1}$ Martha Konieczkowski, ${ }^{1}$ Laura Barisoni, ${ }^{2}$ David B. Thomas, ${ }^{2}$ Santhi Ganesan, ${ }^{3}$ Leslie A. Bruggeman, ${ }^{1}$ Matthias Buck, ${ }^{4}$ and John R. Sedor ${ }^{1,4}$

'Department of Medicine and Rammelkamp Center for Education and Research, MetroHealth System Campus, and ${ }^{2}$ Department of Pathology, University of Miami, Miami, Florida, USA. ${ }^{3}$ Department of Pathology, MetroHealth System Campus, and ${ }^{4}$ Department of Physiology and Biophysics, Case Western Reserve University School of Medicine, Cleveland, Ohio, USA.

APOL1 variants in African populations mediate resistance to trypanosomal infection but increase risk for kidney diseases through unknown mechanisms. APOL1 is expressed in glomerular podocytes and does not vary with underlying kidney disease diagnoses or APOL1 genotypes, suggesting that the kidney disease-associated variants dysregulate its function rather than its localization or abundance. Structural homology searches identified vesicle-associated membrane protein 8 (VAMP8) as an APOL1 protein interactor. VAMP8 colocalizes with APOL1 in the podocyte, and the APOL1:VAMP8 interaction was confirmed biochemically and with surface plasmon resonance. APOL1 variants attenuate this interaction. Computational modeling of APOL1's 3-dimensional structure, followed by molecular dynamics simulations, revealed increased motion of the C-terminal domain of reference APOL1 compared with either variant, suggesting that the variants stabilize a closed or autoinhibited state that diminishes protein interactions with VAMP8. Changes in ellipticity with increasing urea concentrations, as assessed by circular dichroism spectroscopy, showed higher conformational stability of the C-terminal helix of the variants compared with the reference protein. These results suggest that reference APOL1 interacts with VAMP8-coated vesicles, a process attenuated by variant-induced reduction in local dynamics of the C-terminal. Disordered vesicular trafficking in the podocyte may cause injury and progressive chronic kidney diseases in susceptible African Americans subjects.

Conflict of interest: The authors have declared that no conflict of interest exists.

Submitted: January 4, 2017 Accepted: June 13, 2017 Published: July 20, 2017

\section{Reference information:} JCI Insight. 2017;2(14):e92581. https://doi.org/10.1172/jci. insight. 92581

\section{Introduction}

Chronic kidney disease (CKD) is common, affecting more than 20 million Americans, and it is life limiting, even with renal replacement therapy. Advanced CKD disproportionately affect African Americans (1). A landmark discovery associated genetic variation in the $A P O L 1$ gene with excess prevalence of advanced, nondiabetic $\mathrm{CKD}$ in African Americans $(2,3)$. Although data showed higher rates of CKD progression in African Americans, regardless of diabetes status (4), the kidney disease-risk variants failed to associate with diabetic kidney disease $(5,6)$. CKD subjects with $A P O L 1$ risk genotypes benefit less from optimal treatments $(4,7,8)$. Although $A P O L 1$ kidney disease-associated variants are common (12\% of African Americans carry the risk genotype), only a subset of these individuals develop advanced CKD $(2,9)$, consistent with a "second hit" initiating disease that may be resistant to our best available treatments.

APOL1 is the human trypanolytic factor and prevents African sleeping sickness $(10,11)$. An African trypanosome species produces serum resistance-associated (SRA) protein that blocks trypanolysis by inhibiting APOL1 function (11). APOL1-coding polymorphisms, G1 and G2, encode APOL1 proteins that circumvent SRA-dependent resistance and restore parasite killing, but, if present on both alleles, they markedly increase CKD odds in African American patients (2, 3). We and others have shown that APOL1 is localized in human kidney glomeruli (12-14) and that circulating APOL1 levels do not associate with categorical or quantitative CKD phenotypes $(15,16)$. Others have shown that poor kidney allograft outcomes are associated with the APOL1 genotype of the transplanted kidney but not the recipient $(17,18)$. These data suggest that kidney-associated, but not circulating, APOL1 mediates disease. 
Here, we show that APOL1 is synthesized primarily in glomerular podocytes. The abundance and distribution of APOL1 in renal biopsies from patients with risk and nonrisk APOL1 genotypes are similar, suggesting that the G1 and G2 mutations do not change APOL1 localization or trafficking but likely alter its function. Using a bioinformatic structural homology search for SRA, we identified the v-SNARE vesicle-associated membrane protein 8 (VAMP8) as a putative ligand for APOL1 and support this premise with empirical data that show that APOL1 directly interacts with VAMP8, an interaction that is attenuated by the kidney disease risk variants. We propose that APOL1 detects and contains environmental danger signals carried by vesicles marked by VAMP8. Variant APOL1 proteins fail to do so, permitting kidney disease progression.

\section{Results}

APOL1 localization does not vary with disease diagnosis or risk genotype status. Careful phenotyping of gene expression patterns in situ in normal tissue and biopsy specimens can provide clues to pathogenetic mechanisms. We and others have previously shown that the overall fluorescence intensity of APOL1 signal in the glomeruli of focal segmental glomerulosclerosis (FSGS) and HIV-associated nephropathy (HIVAN) samples was diminished relative to glomeruli from samples without renal pathology $(12,14)$. In this study, we further characterized APOL1 localization and distribution in podocytes to determine if these phenotypes were dependent upon kidney disease diagnosis or APOL1 genotype. First, we compared APOL1 distribution in biopsies of diabetic kidney disease, which is not associated with APOL1 risk variants $(5,6)$, to its distribution in biopsies of $A P O L 1$-associated kidney diseases (i.e., hypertension-associated, disease, FSGS and HIVAN) by quantifying APOL1 proximity to the podocyte plasma membrane protein GLEPP1 (Supplemental Table 1 and Supplemental Figure 1, A-J; supplemental material available online with this article; https://doi.org/10.1172/jci.insight.92581DS1). In healthy kidney, APOL1 and GLEPP1 were almost always associated, but APOL1 staining was lost in GLEPP1-positive podocytes in kidney biopsy samples from individuals with kidney disease. However, we did not identify a significant difference in the distribution of APOL1 based upon disease pathology, suggesting that these changes in APOL1 distribution and abundance reflect a generalized response to kidney injury and are not unique to APOL1-associated kidney diseases. In addition, pixel proximity of APOL1 and GLEPP1 was similar in glomeruli of HIVAN biopsies when stratified by $A P O L 1$ risk genotype status. (Supplemental Figure $1 \mathrm{~K}$ ), demonstrating that changes in expression pattern are independent of $A P O L 1$ genotype.

To determine if APOL1 variants alter the intracellular distribution of APOL1, epitope-tagged reference (G0) and variant APOL1 (G1 or G2) constructs were cotransfected into COS7 cells. To model a "second hit," cells were treated with rapamycin to stimulate autophagy, with chloroquine to block autophagic maturation, with oligomycin to inhibit ATP-synthase and mitochondrial function, with thapsigargin to induce endoplasmic reticulum stress, or with lipopolysaccharide to induce cytokine activation. Reference and variant APOL1s colocalized at baseline and after cell stress induction (Supplemental Figure 2). Taken together, we were unable to detect differences in the localization of APOL1 in kidney sections based upon disease pathology or APOL1 genotype or based upon cell culture under tested stress conditions, suggesting that the variants may not impart pathogenicity through dysregulated localization.

APOL1 is synthesized in the podocyte and proximal tubule. Our previous work has shown that the APOL1 protein can be detected in normal human kidney and is localized to the podocyte, proximal tubule, and nonglomerular endothelium (12). Since APOL1 is known to be abundantly expressed in the circulation, we used in situ hybridization to ascertain whether APOL1 is endogenously synthesized in the renal cell types where it was localized or if it is taken up from an extracellular source. These results demonstrate that $A P O L 1$ transcripts are detectable within the podocyte and much less so in some but not all tubules, which we have previously characterized as proximal (12). The abundance of APOL1 transcripts in proximal tubules did not correlate with the robust APOL1 protein abundance detected by immunostaining (Figure 1 and Supplemental Figure 3), suggesting that APOL1 may be taken up from urine or circulation.

We recognized that the staining patterns of APOL1 in the podocyte and proximal tubules were distinct. In the podocyte, the pattern appeared to be homogenous with some puncta, whereas, in the proximal tubule, the pattern appeared distinctly punctate with a basolateral distribution (12). Therefore, we used confocal immunofluorescent microscopy to costain human kidney sections with antibodies against APOL1 and the $\beta$ subunit of ATP synthase, a mitochondrial marker. We found that APOL1 colocalized with mitochondrial markers in the proximal tubule but not in the podocyte (Figure 2, A-C, and Supplemental Figure 4A). To confirm these findings, we performed immunogold electron microscopy and quantified numbers of gold particles, adjusted for 


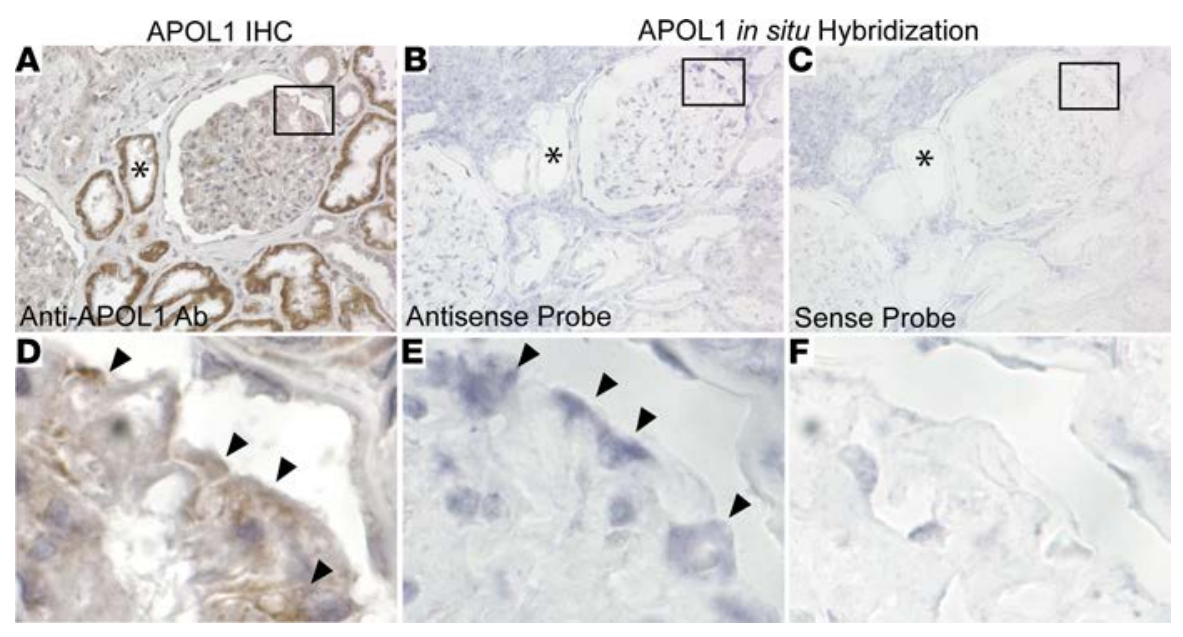

Figure 1. Apolipoprotein L1 mRNA in a normal human kidney by in situ hybridization. (A-C) Representative images from normal human kidney tissue obtained from two tumor nephrectomies. Serial sections showing apolipoprotein L1 (APOL1) expression by immunohistochemistry (A) (color reaction product is brown, counterstained with hematoxylin) and with in situ hybridization using antisense probe (B) (color reaction product is blue, with no counterstain) and sense probe as control (C). APOL1 protein and mRNA expression is present in the glomerulus but in contrast to the strong positive signal (denoted by asterisks) detected by immunohistochemistry (A); a weak in situ hybridization signal is seen in some but not all proximal tubules (B). (D-F) Magnification of boxed regions in $\mathbf{A}-\mathbf{C}$ shows that a positive signal is present in podocytes (arrowheads) for both staining methods. Original magnification, $\times 40(\mathbf{A}-\mathbf{C}) ; \times 285(\mathbf{D}-\mathbf{F})$. podocyte region of interest and area. APOL1 was labeled in the cytosol and contiguous to cytoskeletal elements (Figure 2, D and G, Supplemental Figure 5A, and Supplemental Figure $4, \mathrm{~B}$ and $\mathrm{C}$ ) and vesicles (Figure 2, E and H). APOL1 localized to proximal (Figure 2, $\mathrm{F}$ and I) but not distal tubular (Supplemental Figure 5, B and C) or podocyte mitochondria (Supplemental Figure 4, B and E). Additionally, significantly fewer gold particles, adjusted for surface area analyzed, were counted in tissue sections that were incubated with rabbit IgG compared with kidney sections incubated with anti-APOL1 (Supplemental Figure 6). In normal human kidney sections, APOL1 also did not colocalize with lysosomal marker LAMP1 in podocytes and proximal tubules (Supplemental Figure 7).

APOL1 interacts with VAMP8. Since APOL1 expression levels and localization did not vary with $A P O L 1$ risk genotype, we hypothesized that APOL1 may have an unrecognized cellular function that is dysregulated by the kidney disease-associated variants by altering an interaction with a specific binding partner(s). The amino-terminal $\alpha$-helical domain of SRA (aa 31-79) directly interacts with the APOL1 carboxy terminal domain (aa 339-398); the interaction is lost with variant APOL1-G2 (2, 11). No human proteins with plausible homology to the primary sequence of aa 31-79 of the SRA protein were identified. Since secondary structure homology can be conserved when the primary sequence has diverged, we used an online structural homology search program to identify human proteins structurally similar to the APOL1-interacting domain of trypanosomal SRA and identified the SNARE protein VAMP8, as a top hit (Supplemental Table 2); several other SNARE proteins also were identified as structural homologs.

To empirically demonstrate the APOL1:VAMP8 interaction, epitope-tagged, full-length APOL1 and VAMP8 constructs were cotransfected into 293T cells. VAMP8 precipitated full-length APOL1 but not APOL1 lacking the SRA-binding domain (APOL1- $\triangle$ aa 339-398) (Figure 3A). Using confocal immunofluorescence microscopy, APOL1-GFP and VAMP8-myc-FLAG also demonstrated substantial colocalization in a vesicular pattern in HeLa cells (Figure 3B). Further, APOL1 was colocalized with VAMP8 in glomeruli within GLEPP1-positive podocytes (Figure 3, C and E) but not in proximal tubules (Figure 3D). In contrast to APOL1-G0, the interaction between APOL1-G1 or -G2 and VAMP8 was attenuated (Figure 4A). Pull-down experiments using His-tagged APOL1-G0, -G1, and -G2 and myc-FLAG-tagged VAMP8 confirmed the results of the coprecipitation experiments (Figure 4B). Surface plasmon resonance was used to further characterize this interaction. APOL1 (residues 305-398), with an N-terminal GST tag that increased protein solubility, was immobilized on a biosensor chip. VAMP8 (aa 1-76), which encompasses the SNARE domain (residues 12-72) and the region with structural homology to SRA, was the analyte. A dose-responsive binding of VAMP8 with estimated $\mathrm{Kd}$ of 100 to $300 \mu \mathrm{M}$ was demonstrated for APOL1-G0 (Figure 4C and Supplemental Figure 8), which was markedly attenuated in case of the variants (Figure 4, D-F, and Supplemental Figure 8). To validate our structure-based prediction of APOL1 interaction with SNARE proteins, we tested interaction of APOL1 with another R-SNARE, VAMP1 (Supplemental Table 2). APOL1-G0-GFP interacted with VAMP1-myc-FLAG when overexpressed in 293T cells (Figure 4A).

APOL1 variants are helix stabilizing. Three-dimensional structures of APOL1 or other APOL family proteins have not been resolved experimentally. Computational modeling and molecular dynamics (MD) simulations have been utilized in the past to model the tertiary structure of various domains of APOL1 (10, 19, 20). We utilized threading programs (21) that identify suitable template proteins from solved structure databases to model the 3-dimensional structure of the C-terminal domain of APOL1. The secondary structure 

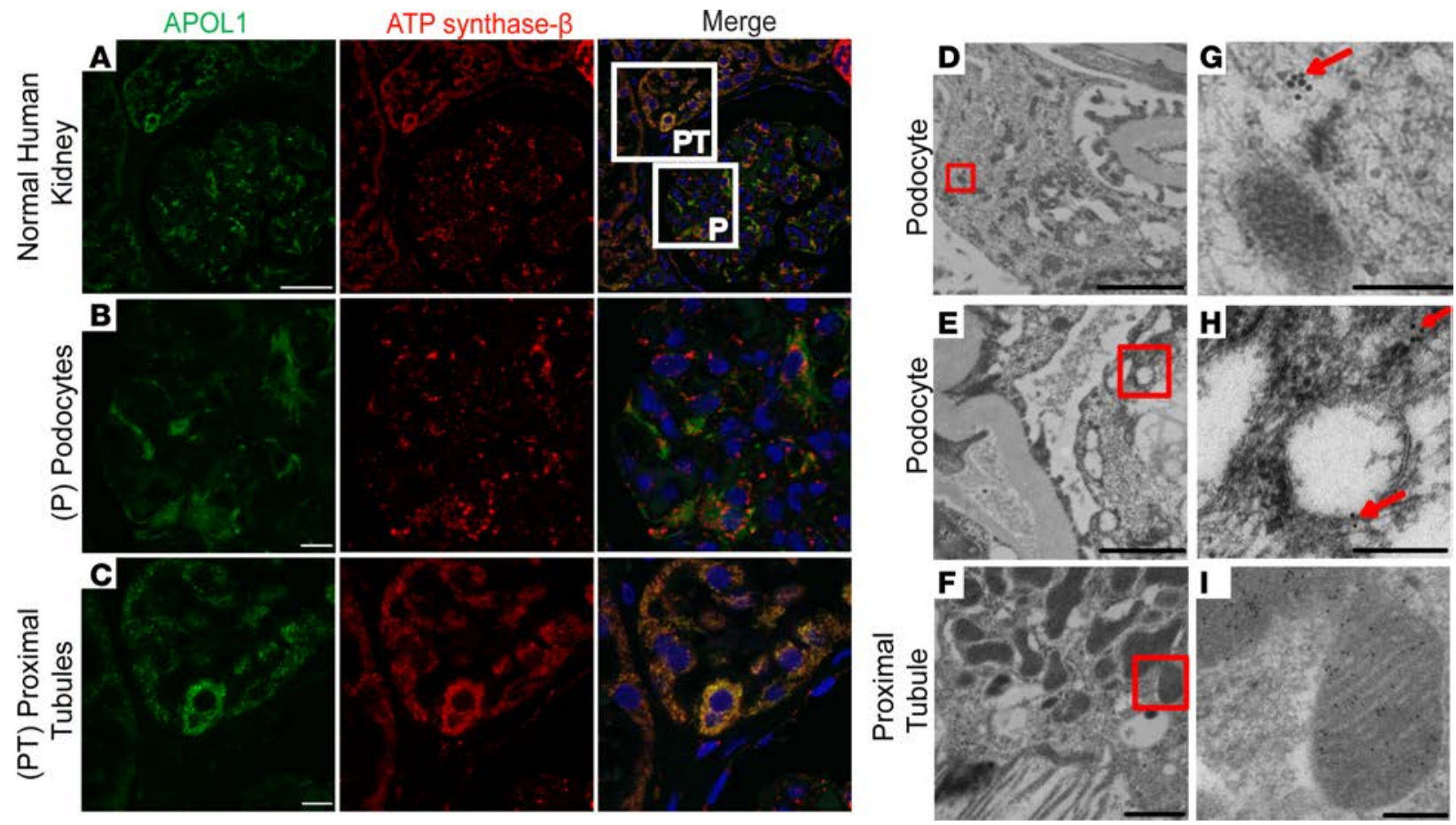

Figure 2. Subcellular localization of apolipoprotein L1 in normal human kidney. (A-C) Confocal immunofluorescence microscopy images showing apolipoprotein L1 (APOL1) (green) colocalizing with the $\beta$ subunit of ATP synthase (red) in proximal tubules of normal human kidneys (C) but not in podocytes (B). (B and $\mathbf{C}$ ) Magnified images of the boxed regions shown in the merged image of $\mathbf{A}$, showing the podocyte (B) and the proximal tubule (C). Nuclei were stained with TOTO-3 iodide (scale bars: $10 \mu \mathrm{m}$ ). (D-F) Immunogold electron microscopy image of human podocyte and proximal tubules from a normal human kidney (scale bars: $2 \mu \mathrm{m}$ ). (G-I) Magnification of the boxed regions from D-F demonstrates (G) gold particles in cytoplasm associated with podocyte cytoskeleton (red arrow), (H) vesicles in podocytes (red arrows), and (I) gold particles in proximal tubules localizing to mitochondria (scale bars: $0.2 \mu \mathrm{m}$ ).

of the APOL1 C-terminal region is predicted to have an $\alpha$-helical conformation. The computationally modeled 3-dimensional structure of APOL1 (residues 305-398) consists of $3 \alpha$-helices (H1, H2, and H3) folded as a bundle and linked by short loops (Figure 5A and Supplemental Figure 9). We used multiple classical all-atom MD simulations with multiple random seeds to understand the differences in the conformational and dynamical behavior of the C-terminus of the APOL1 variants compared with the wild-type protein (Supplemental Table 3). The initial computationally modeled structures of APOL1-G0 and variants were similar after a brief minimization (Figure 5A); however, the structures diverged markedly within $40 \mathrm{~ns}$ of $\mathrm{MD}$ simulation and showed different amplitudes of internal dynamics (Figure 5B and Supplemental Figure 10). Root mean square deviation of the $\mathrm{C} \alpha$ atoms measures the deviation from the starting structures as a function of simulation time (Figure 5C and Supplemental Figure 10, A, E, and G). Overall, the simulation demonstrated that the conformation of G1 and G2 variants remained relatively unchanged, whereas the reference (G0) structure diverged from its starting conformation. Compared with the variants, increased amplitude of the dynamics for the wild-type structure was evident from the root mean square fluctuation (RMSF) of $\mathrm{C} \alpha$ atoms, calculated over the last $10 \mathrm{~ns}$ of the simulations. Residues of the G0 C-terminus, predominantly in the $\mathrm{H} 1$ and $\mathrm{H} 3$ helices as well as the connecting loops, showed higher flexibility compared with the variants, as demonstrated by the higher RMSF values (Figure 5D and Supplemental Figure 10I). The collective dynamics of protein motion was examined by a principal component analysis of the MD trajectory. The projections of MD trajectory on the first three principal components show the higher flexibility of the G0 C-terminus compared with the same region of the variants. In addition, substantial overlap was seen between the two variants in the manner in which they sample conformational space compared with G0 (Figure 5, E and F; Supplemental Figure 10, B and F; and Supplemental Figure 11H). The projection of the first eigenvector onto the initial model also confirmed the larger flexibility of $\mathrm{H} 1$ and $\mathrm{H} 3$ in G0, which favors an "open" conformation (Supplemental Figure 10, C and D). Similar results were obtained when MD simulations were carried out on a model of the C-terminal region of APOL1 that interacts with SRA (compared with the above-mentioned structure, the protein is shorter at the $\mathrm{N}$-terminus comprising aa 
A

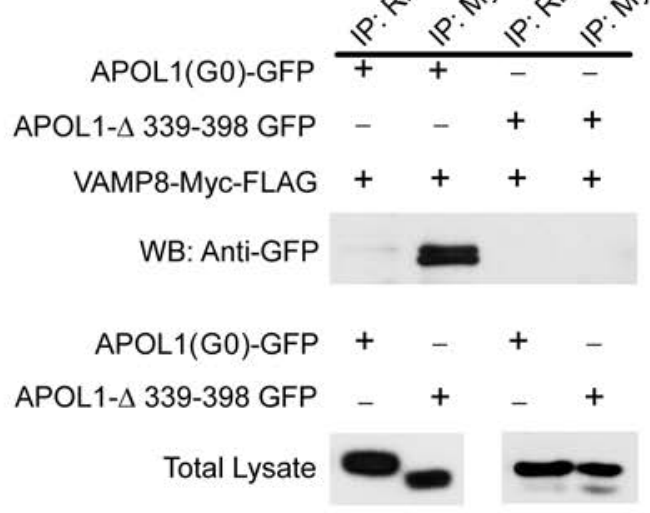

WB: Anti-GFP Anti-FLAG

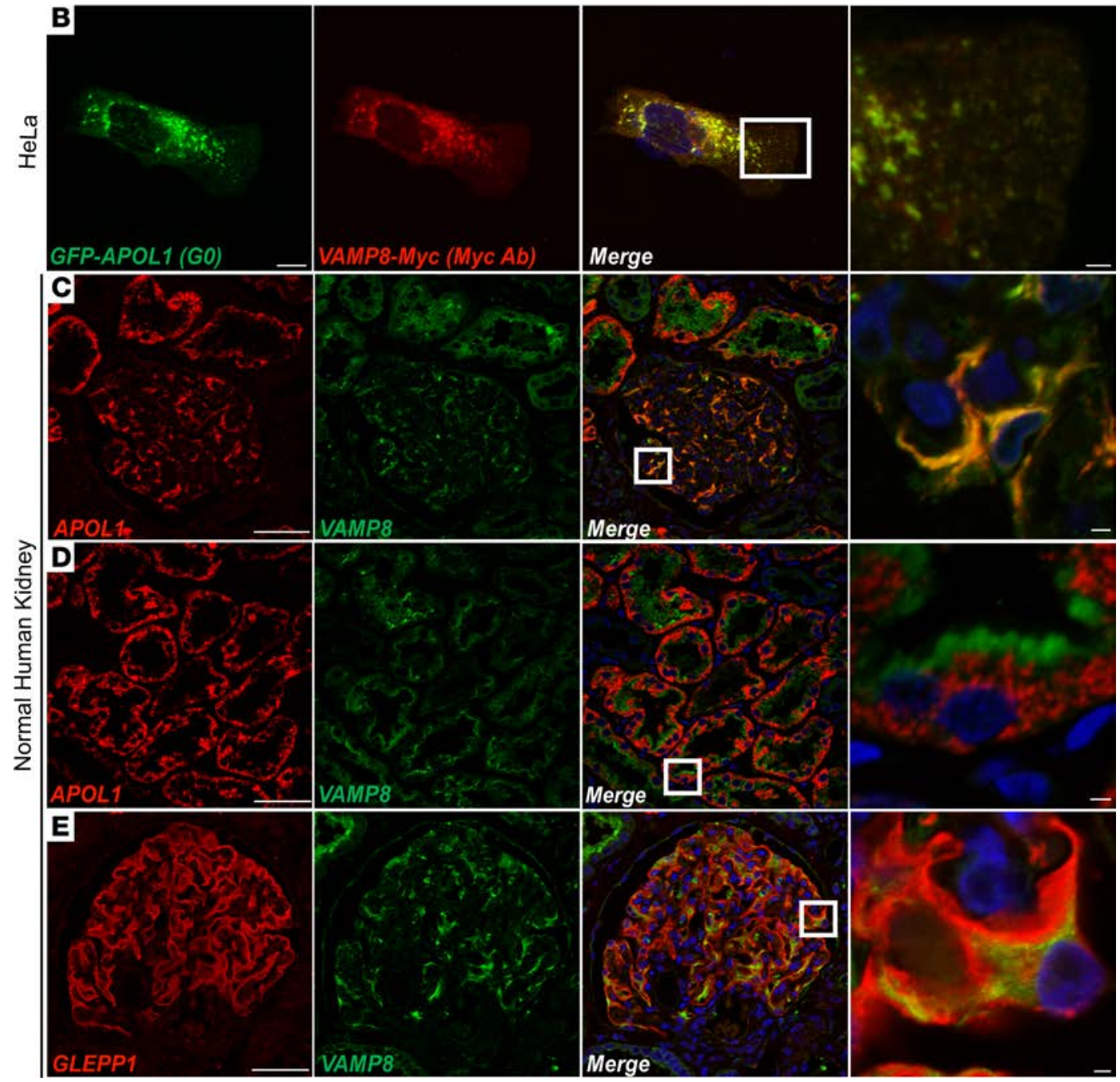

Figure 3. Apolipoprotein L1 interacts with vesicle-associated membrane protein 8 and colocalizes in vitro and in podocytes of human kidney. (A) Representative Western blot of immunopreciptates and whole cell lysates from 293T cells transfected with myc-FLAG-tagged vesicle-associated membrane protein 8 (VAMP8) and carboxy terminal GFP-tagged apolipoprotein L1 (APOL1) plasmids as shown. Full-length GFP-tagged APOL1-G0, but not APOL1 lacking the trypanosomal serum resistance-associated (SRA) protein-interacting domain (aa 339-398), coimmunoprecipitated with myc-FLAG-tagged VAMP8 using an anti-Myc antibody. Rabbit IgG was used for control immunoprecipitation experiments $(n=3)$. (B) Transiently expressed GFP-tagged APOL1-GO (green) and myc-FLAG-tagged VAMP8 (red) colocalize in a vesicular pattern in HeLa cells (scale bars: $10 \mu \mathrm{m})$. (C) Endogenous APOL1 (red) and VAMP8 (green) colocalize in podocytes of normal human kidney (scale bars: $50 \mu \mathrm{m}$ ). (D) APOL1 (red) in basolateral domains of proximal tubules does not colocalize with VAMP8 (green), which is present in an apical distribution (scale bars: $50 \mu \mathrm{m}$ ). (E) Localization of VAMP8 (green) within glomerular podocytes was confirmed by costaining with glomerular epithelial protein 1 (GLEPP1) (red), a podocyte marker, in normal human kidney (scale bars: $50 \mu$ m). VAMP8 staining was also detectable in other glomerular cell types. In all images, nuclei were stained with TOTO-3 iodide (scale bars: $2 \mu \mathrm{m}$ [inset]). 
A

\begin{tabular}{|c|c|c|c|c|c|c|}
\hline & IP: N & & & & $: \mathrm{Rb}$ & $0 \lg G$ \\
\hline APOL1(G0)-GFP & $+\quad-$ & - & + & + & - & -+ \\
\hline APOL1(G1)-GFP & $-\quad+$ & - & - & - & + & - \\
\hline APOL1(G2)-GFP & $-\quad-$ & + & - & - & - & + \\
\hline VAMP8-Myc-FLAG & $+\quad+$ & + & - & + & + & +- \\
\hline VAMP1-Myc-FLAG & $-\quad-$ & - & + & - & - & -+ \\
\hline WB: Anti-GFP & $\infty$ & & - & & & \\
\hline $\begin{array}{r}\text { Total Lysate } \\
\text { WB: Anti-GFP }\end{array}$ & $-m$ & $=$ & 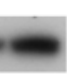 & & & \\
\hline $\begin{array}{c}\text { Total Lysate } \\
\text { WB: Anti-Myc }\end{array}$ & & & & $\begin{array}{l}4 V A \\
4 V A\end{array}$ & MPE & $\begin{array}{l}\text { 1-Myc } \\
\text { 8-Myc }\end{array}$ \\
\hline
\end{tabular}

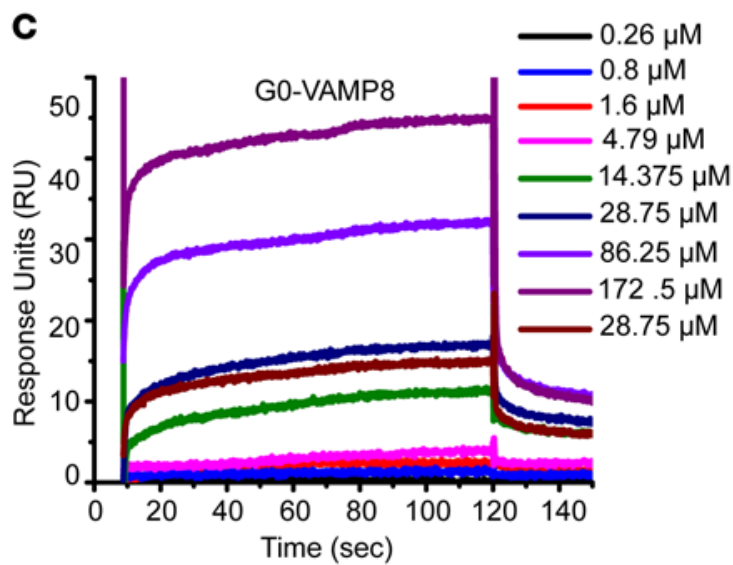

E

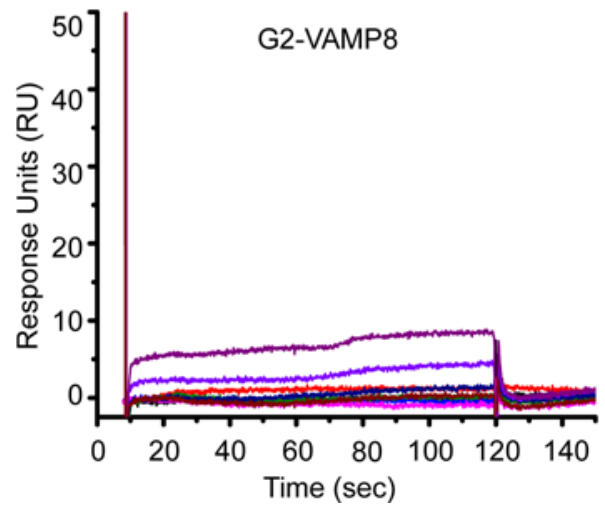

B

\begin{tabular}{rlllll} 
& \multicolumn{3}{c}{ +VAMP8-Myc-FLAG } \\
\cline { 2 - 6 } NHE1-His & + & - & - & - \\
APOL1(G0)-His & - & + & - & - \\
APOL1(G1)-His & - & - & + & - \\
APOL1(G2)-His & - & - & - & + \\
Input & & & & & \\
Coomassie stain
\end{tabular}

WB: FLAG

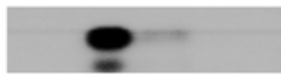

D

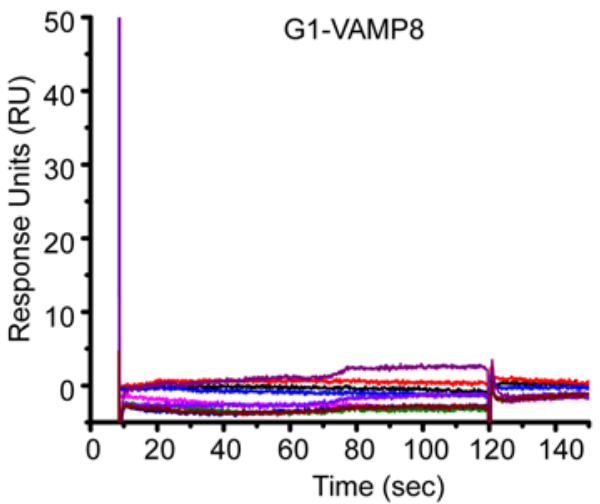

$\mathbf{F}$

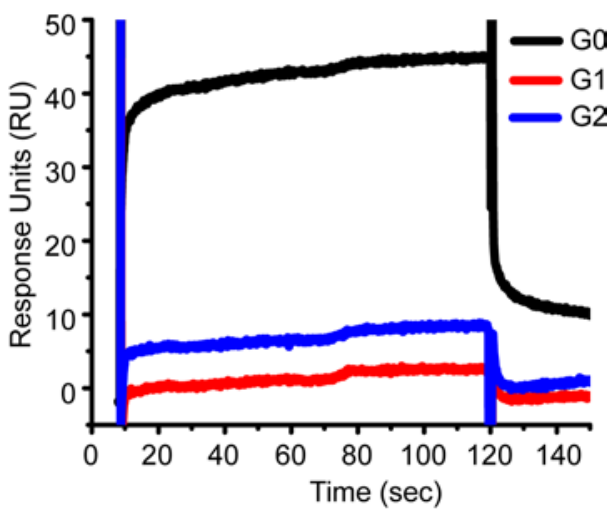

Figure 4. Kidney disease-associated apolipoprotein L1 variants attenuate protein interaction with vesicle-associated membrane protein 8. (A) Carboxy terminal GFP-tagged apolipoprotein L1 (APOL1) (GO, G1, or G2) were overexpressed with carboxy terminal myc-FLAG-tagged vesicle-associated membrane protein 8 (VAMP8) in 293T cells and were coimmunoprecipitated with VAMP8 using an anti-myc antibody. Coimmunoprecipitated APOL1 was detected by Western blot analysis using anti-GFP antibody. The efficiency of VAMP8 coimmunoprecipitation with the APOL1-G1 and -G2 risk variants was reduced compared with reference sequence APOL1-GO. C-terminal GFP-tagged APOL1-GO also interacts with vesicle-associated membrane protein 1 (VAMP1) as shown by coimmunoprecipitation by C-terminal myc-FLAG-tagged VAMP1. Rabbit IgG was used for control immunoprecipitation experiments ( $n=4$ for $\mathrm{GO}$ and $n=3$ for G1 and G2). (B) Carboxy terminal 6X-His-tagged APOL1 proteins (G0, G1, or G2) or His-tagged sodium/hydrogen exchanger 1 (NHE1), as a nonspecific control protein, on Talon beads were incubated with cell lysates from 293T cells overexpressing myc-FLAG-tagged VAMP8. Representative Western blot of bead-bound protein using anti-myc antibody demonstrates diminished interaction of APOL1-G1 and -G2 variants with VAMP8 $(n=2)$. (C-F) Surface plasmon resonance analysis of VAMP8 (residues 1-76) with amino-terminal 6X-His and T7 tag binding to immobilized N-terminal GST-tagged APOL1 proteins (G0, G1, or G2). GST-tagged APOL1 proteins (residues 305-398) were immobilized on the biosensor chip by amine coupling, and purified VAMP8 was injected at varying concentrations, as labeled. Sensograms show a concentration-dependent binding of VAMP8 to the carboxy terminal domains of APOL1-GO (C), whereas, the interaction of VAMP8 with the carboxy terminus of the variants APOL1-G1 (D) and APOL1-G2 (E) is diminished. (F) The injection of VAMP8 at a single concentration (172.5 $\mu$ M) shows reduced binding to the APOL1-G1 and APOL1-C2 variants compared with the APOL1-G0 reference sequence ( $n=4$ for G0 and $n=3$ for G1 and G2). 
A

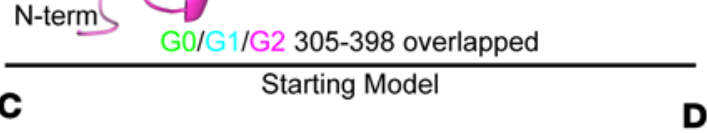

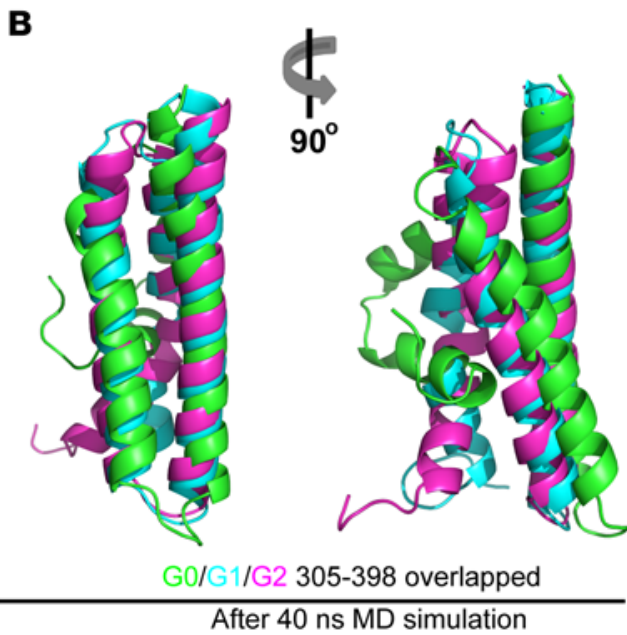

D

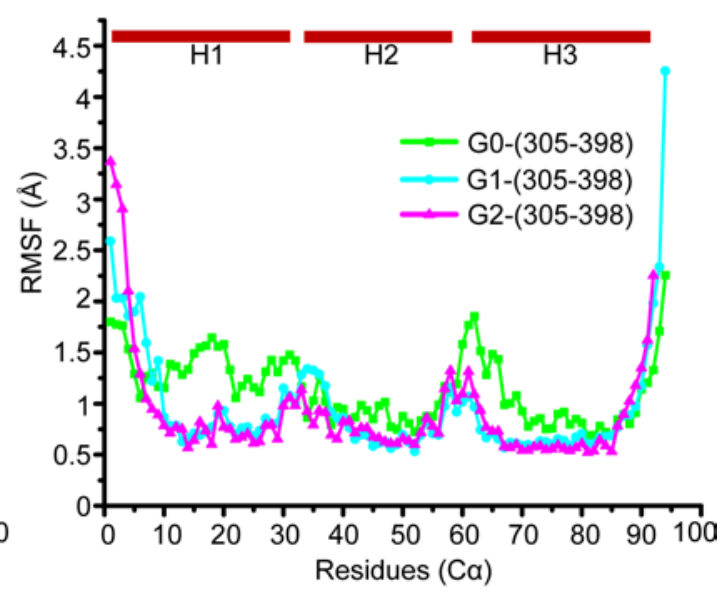

$\mathbf{F}$

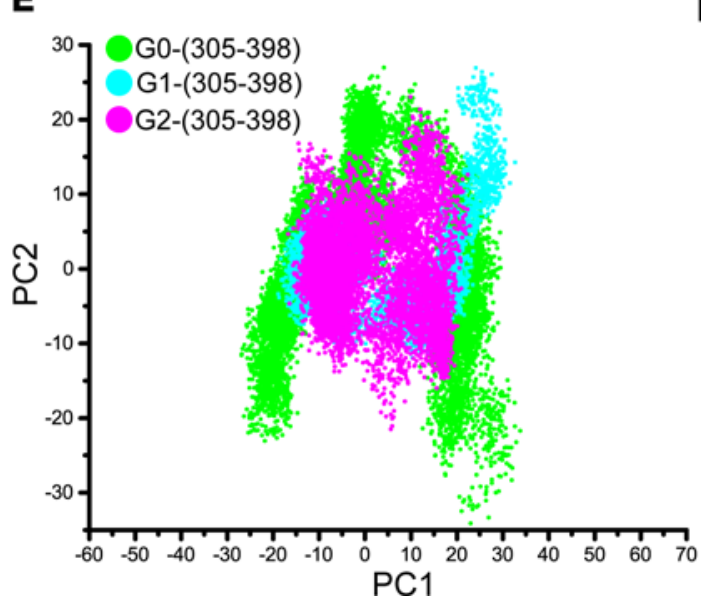

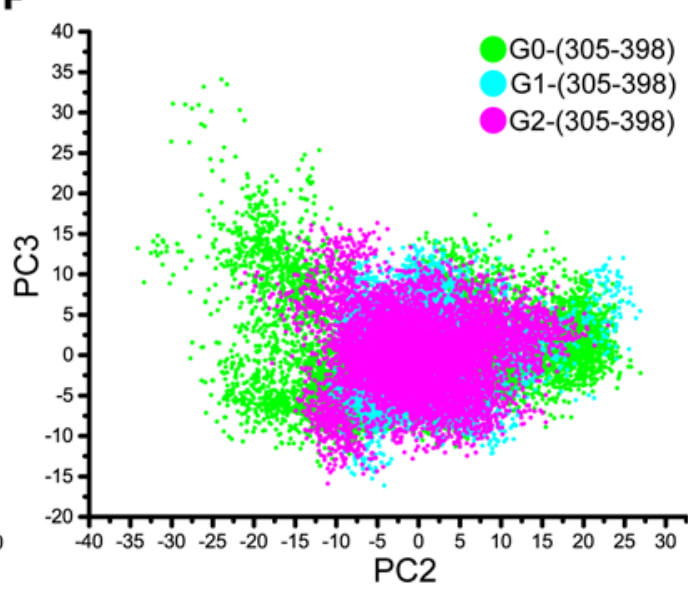

Figure 5. Kidney disease-associated variants are more compatible with a helix-bundle ("closed") structure compared with the wild type protein. (A) The 3-dimensional structure of the apolipoprotein L1 (APOL1) carboxy terminal region (aa 305-398) was modeled by threading using Iterative Threading ASSEmbly Refinement (I-TASSER). Structures of APOL1-GO (green), -G1 (cyan), and -G2 (magenta) are overlapped showing similar starting structures. The predicted structure of the carboxy terminal domain has $3 \alpha$-helices $(\mathrm{H} 1, \mathrm{H} 2$, and $\mathrm{H} 3)$ connected by loops. The magnified view demonstrates the position of residues S342 and 1384, which are altered in APOL1-G1, and residues N388 and Y389, which are deleted in APOL1-G2. (B) After 40 ns of molecular dynamics (MD) simulations, the final structures were superimposed to show differences in the wild-type and risk variant structures as they evolved during the simulations. (C) Root mean square deviation (RMSD) of $\alpha$ carbon ( $C \alpha$ ) atoms relative to the coordinates of initial structure as a function of simulation time shows higher deviation of the APOL1-G0 structure compared with the APOL1-G1 and -G2 variants. (D) Flexibility of backbone C $\alpha$ atoms, indicated by root mean square fluctuation (RMSF) on the $y$ axis for each residue shown on the $x$ axis. The results represent mean RMSF values in the last $10 \mathrm{~ns}$ of two separate MD simulations carried out for $40 \mathrm{~ns}$. Backbone atoms of APOL1-GO show increased flexibility, as demonstrated by the higher RMSF values compared with the variant proteins, especially in $\mathrm{H} 1$ and $\mathrm{H} 3$. (E and F) Projection of MD trajectories (40 ns) of the APOL1-GO, - G1, and - G2 carboxy terminal domain (aa 305-398) on the first, second, and third principal components (PC) derived from C $\alpha$-only principal component analysis, showing close clustering of the APOL1-G1 and -G2 conformations compared with APOL1-GO. 
A

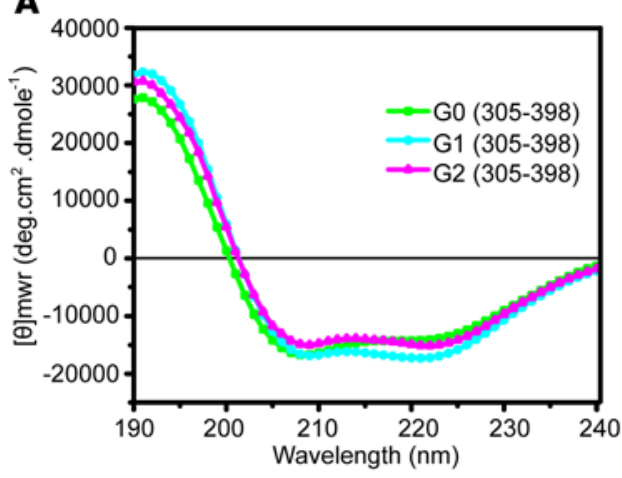

B

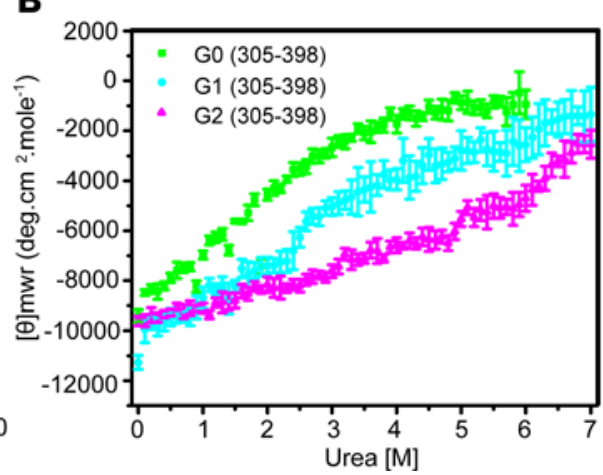

C

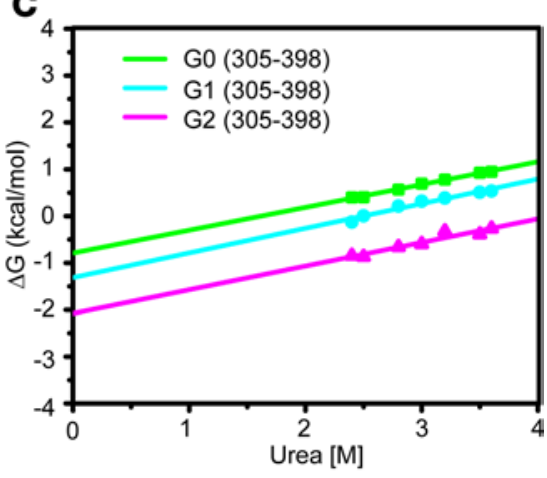

Figure 6. Secondary structure analysis of the C-terminal domain of apolipoprotein L1 by circular dichroism spectroscopy. (A) Far UV circular dichroism (CD) spectra of APOL1-GO (green), -G1 (blue), and -G2 (red) carboxy termini (aa 305-398) showing predominant $\alpha$-helical conformation. The mean residue ellipticity ( $\theta$ mwr) is plotted on $y$ axis versus wavelength $(n m)$ on the $x$ axis (GO $n=3, G 1 n=2, G 2 n=2$ ). (B) Representative experiment showing mean residue ellipticity of reference and variant apolipoprotein L1 (APOL1) carboxy termini as a function of increasing urea concentration in $50 \mathrm{mM}$ sodium sulfate, $20 \mathrm{mM}$ potassium phosphate, $\mathrm{pH}=7.0$, at $37^{\circ} \mathrm{C}(\mathrm{C} O \mathrm{n}=3, \mathrm{G} 1 n=2, \mathrm{G} 2 n=2)$. Data points at each step increase in urea concentration were averaged over 10 recordings and are shown as mean $\pm S E M$. (C) Estimation of free energy of folding $\left(\Delta \mathrm{G}_{0}\right)$ from changes in $C D$ as a function of denaturant. The experimental values of $\Delta \mathrm{G}_{i}$ corresponding to the ith concentration of urea were calculated in the transition region to obtain a linear plot (42). The free energy of folding corresponds to the $y$ intercept.

339-398 and includes the sites of mutation(s) in both variants). Although the initial models generated by threading were similar for the wild type and variant structures (Supplemental Figure 11, A and B), a 40-ns MD simulation showed uncoiling of G0, whereas the variant proteins maintained a stable helical conformation (Supplemental Figure 11, C and D). Again, the RMSF analysis revealed higher flexibility of G0 residues in $\mathrm{H} 3$ helix and interconnecting loop compared with variants (Supplemental Figure 11E). Close clustering of conformational space sampled by G1 and G2 variants was seen in the principal component analysis of molecular motions, compared with the reference protein (Supplemental Figure 11F). Taken together, these results suggest that relative to $\mathrm{G} 0$, the $\mathrm{G} 1$ and $\mathrm{G} 2$ variants of APOL1 are more compatible with the folded helix-bundle conformation in the carboxy terminal domain of APOL1.

To experimentally confirm the helix-stabilizing properties of G1 and G2 variants compared with G0, we analyzed the secondary structure of the APOL1 C-terminal region (residues 305-398) using circular dichroism (CD) spectroscopy. As predicted by computational methods, the secondary structure of the APOL1 C-terminus showed a predominance of the $\alpha$-helical structure. The $\alpha$-helical composition of the C-terminal region did not differ significantly in G1 and G2 variants compared with the reference protein at baseline (Figure 6A and Supplemental Table 5). We then measured the stability of the C-terminal helix of reference and variant APOL1 by monitoring the change in ellipticity in response to increasing concentration of urea (Figure 6B), a method generally preferred to thermal denaturation. The free energy of folding $\left(\Delta \mathrm{G}_{0}\right)$ calculated from the unfolding experiments showed higher conformational stability of the G1 and G2 C-terminal helix compared with the reference protein (Figure 6C and Table 1).

To understand the structural basis of the interaction of APOL1 and VAMP8, we generated a computational model of the APOL1 C-terminal region and the VAMP8 SNARE domain complex. The C-terminal region of APOL1 (residues 339-398) and the SNARE domain of VAMP8 (residues 12-68) were modeled on the crystal structure of endosomal SNARE (PDB ID: 1GL2) (Supplemental Figure 12), which shows a 4-helix coiled-coil bundle in a parallel orientation. APOL1 G0 C-terminal region/ VAMP8 SNARE domain stoichiometry was set to $3: 1,1: 3$, or 1:1, and the structures were then subjected to a 15-ns MD simulation (Supplemental Table 4). The APOL1 G0/VAMP8 SNARE domain interaction is plausible with a 3:1 stoichiometry, while a 1:3 interaction or a monovalent (1:1) complex appeared unfavorable (Supplemental Figure 12, A-C). Helix 3 (H3) of the APOL1 C-terminus appears to predominantly mediate this interaction (Supplemental Figure 12A). Analysis of models of the 3:1 APOL1 G0/VAMP8 complex from three independent simulations showed that the quaternary structure had a hydrophobic core that was further stabilized by external interhelix polar contacts (Supplemental Figure 12 , D-G). The polar contacts formed between helices were reproducible during simulations performed using random seeds (Supplemental Figure 12, E-G). Importantly, residues constituting the G1 and G2 variants were not involved significantly in the formation of stabilizing contacts. 
Table 1. Free energy of folding $\left(\Delta \mathrm{G}_{0}\right)$ of APOL1 carboxy terminus (aa 305-398)

\begin{tabular}{lcc}
\hline Sample & $\boldsymbol{\Delta G}_{\mathbf{0}}(\mathrm{kcal} / \mathbf{m o l})$ & $\boldsymbol{m}(\mathbf{k c a l} / \mathbf{m o l} / \mathbf{M})$ \\
G0 (305-398) & $-0.78 \pm 0.05$ & 0.488 \\
G1 (305-398) & $-1.31 \pm 0.13$ & 0.526 \\
G2 (305-398) & $-2.08 \pm 0.20$ & 0.504
\end{tabular}

Data in Figure 6C were fitted to line $y=m x+b$, where $y$ is the free energy ( \pm standard error) at each concentration of urea, $x$ is the urea concentration, $m$ is the slope, and $b$ is the $y$ intercept, which represents the free energy in the absence of denaturant.

\section{Discussion}

Building upon our previous results (12), we have further characterized the localization and expression patterns of APOL1 in normal human kidney samples in biopsies from APOL1-associated kidney diseases and diabetic kidney disease, which does not associate with $A P O L 1$ variants $(5,6)$. The APOL1 protein localizes to the podocyte, proximal tubules, and extraglomerular vascular endothelium in the absence of disease. APOL1 mRNA transcripts are detectable in the podocyte and at lower intensity in proximal tubule cells. Higher intensity staining of APOL1 protein in proximal tubules by immunohistology contrasts with the weaker $A P O L 1 \mathrm{mRNA}$ signal in in situ hybridization. APOL1 in the tubules could partly be imported from the circulation or urine. However, circulating levels of APOL1 do not associate with categorical or quantitative CKD phenotypes $(15,16)$, and others have shown that poor kidney allograft outcomes are associated with the APOL1 genotype of the transplanted kidney and not the recipient $(17,22)$. Together, these results suggest that APOL1 synthesized in the podocyte mediates the pathogenesis of APOL1-associated kidney diseases.

Alterations in the expression pattern of APOL1 in the setting of APOL1-associated kidney disease include reduced protein abundance in the podocyte and proximal tubule as well as APOL1's appearance in the vascular media of arterioles and small arteries in the kidney. Our results again found these differences in the APOL1 localization pattern but show that they are not restricted to APOL1-associated diseases. These results suggest that the reduction in the protein abundance of APOL1 in the presence of disease may be a response to kidney damage and therefore is less likely to be a central feature in the pathogenesis of APOL1-associated disease. We have previously demonstrated reduced podocyte density in mice transgenic for APOL1-G2 compared with wild-type mice or mice transgenic for APOL1-G0 (23). The reduction of APOL1 staining in glomeruli we evaluated might reflect podocyte loss, but the experimental design did not allow quantification of podocyte number stratified by genotype. Whether differential expression of variant APOL1s cause reduced podocyte density, which predisposes to progressive CKD after a "second hit," needs further investigation in a larger human data set. Further in vitro experiments demonstrating similar subcellular localization of APOL1-G0 and -G2 variants in the presence and absence chemical cell stress suggest that alteration in protein trafficking related to the APOL1 risk variants is not mediating disease pathogenesis. Together, these results suggest that the risk variants are more likely altering a functional role of APOL1, as opposed to its cellular distribution or subcellular localization. In contrast to these results, another study showed that variant APOL1s had increased localization with RAB7- and RAB11-labeled endosomes compared with G0 in transiently transfected HEK293 cells and cultured human podocytes (24).

A bioinformatic search for proteins with structural homology to the N-terminal amphipathic helix of the trypanosomal SRA protein, the only known protein interactor with APOL1, was enriched in R-SNARE proteins (11). The interaction of APOL1 with VAMP8 was confirmed biochemically and was supported by microscopy demonstrating colocalization in the podocyte of human kidney tissue. APOL1-G0 also interacted with another R-SNARE protein, VAMP1, which had a high score on the bioinformatic search (Supplemental Table 2). Although the coiled-coiled SNARE domains are highly conserved among R-SNARE proteins, amino acid variations determine trafficking specificity (25). Our data suggest that APOL1 could form part of a multiprotein complex by mediating selective assembly of SNARE proteins. Kidney-disease variant-dependent loss of the APOL1:SNARE protein interaction is consistent with the recessive mode of inheritance that best explains the association between $A P O L 1$ and nondiabetic kidney diseases. Others have proposed a plausible mechanism by which a recessive genotype could generate a gain-of-function phenotype, i.e., a protein oligomer activates pathways deleterious to the cell (26).

Although the APOL1:VAMP8 interaction was confirmed by in vivo experiments, the low affinity of the interaction estimated by an in vitro method (SPR) further suggests the presence of unidentified proteins or specific intracellular milieu favoring the interaction. There are multiple explanations for this observed low affinity of interaction. First, there is evidence that APOL1 inserts into plasma and endosomal membranes, which is at least partially mediated by residues in the C-terminal domain (26-28). A low affinity of interaction can be meaningful in a membrane environment in which local concentration of proteins can be considerably higher compared with other intracellular compartments due to clustering and oligomerization, a scenario that is not modeled in SPR experiments. Second, we believe that stoichiometry is critical for optimal interaction of APOL1 C-terminal domain and VAMP8. SPR measures protein-protein inter- 
actions using an immobilized ligand and does not authentically model intracellular stoichiometry. Third, coprecipitation and pulldown experiments were performed in cells in which the membrane environment would facilitate assembly of protein complexes with proper stoichiometry of constituent proteins.

To better understand the APOL1:VAMP8 interaction, we used computational and biophysical methods. Our model suggests that the C-terminal of APOL1-G0 exists in an equilibrium state between an "open" and "closed" or autoinhibited conformation. The open conformation allows interaction with VAMP8, and, remarkably, the altered amino acid residues found in the G1 and G2 variants are not involved in those contacts. Instead, the G1 and G2 variants stabilize the closed state, and this leads to an inability to interact with VAMP8 in addition to the structurally homologous trypanosomal defense protein SRA. It follows that reversing the dynamics of the variant APOL1 protein to an "open" state, for example, by small molecules that disrupt interactions specific to the closed state, should then restore the normal functional status. Increased conformational stability of a "closed" state has been previously shown using NMR to negatively affect functional protein interactions required to initiate signaling activity (29).

While our project neared completion, a paper was published by Sharma et al. (20), which described a similar study with reference and the variant APOL1 C-terminal fragments. Their study focused on the shorter 2-helix fragment 339-398, which had already been predicted in other studies $(19,30)$. Using CD and $\mathrm{MD}$ simulations, the authors suggest that the variants have less structural stability than the reference. Clearly these results contrast ours, with the 3-helix longer construct 305-398. While our data are not mutually contradictory in principle, we also note that a very old force field was used for the MD simulations in Sharma et al. and that only single simulations were carried out. The CD data show a difference in helical content (which can easily arise if the protein concentrations are not accurate) but - if an off-set were to be corrected - actually demonstrate a similar thermal melting behavior. It is likely that the addition of a third helix in our construct provides additional stability to the closed structure and that this might alter the behavior seen to make it consistent with Sharma et al. (20). A resolution of these differences will need to await the independent structure determination of the constructs by both groups and a further characterization of dynamics of the reference protein and of the two variants.

Our demonstration of a protein interaction between APOL1 with VAMP8 was based upon bioinformatic predictions, and these experiments characterize the APOL1:VAMP8 interaction. Additional candidate interactors were identified using this in silico approach, and the interaction of another SNARE protein, VAMP1, with APOL1-G0 was empirically demonstrated. SNARE proteins were overrepresented among the top hits and, upon confirmation, could also be candidates for mediating the pathogenesis of APOL1-associated kidney diseases. SNARE proteins are known to participate in oligomeric protein complexes to mediate their cellular vesicular trafficking functions and membrane fusion. MD simulations suggest that the APOL1:VAMP8 interaction is most stable in a 3:1 stoichiometry, but this does not preclude the possibility that APOL1 is able to interact with alternative SNARE proteins in the same or different stoichiometries.

The demonstration of an interaction between APOL1 and VAMP8 and the bioinformatic prediction that APOL1 may interact with other SNARE proteins suggest that APOL1 may participate in the vesicular trafficking and membrane fusion pathways. APOL1-dependent mechanisms of trypanolysis involve endolysosomal pathways $(27,31,32)$, and VAMP8 mediates fusion between late endosomes and lysosomes (33). Localization of VAMP8 with its cognate autophagosomal SNARE partner STX17 is diminished in presence of APOL1 variants (24). In addition, mice and Drosophila transgenic for human APOL1 kidney disease risk variants have been shown to have disordered endosomal trafficking and variant-dependent glomerular and nephrocyte injury $(24,34,35)$. Our results suggest a model in which APOL1, in concert with VAMP8 or other SNARE proteins, identifies vesicles containing cargo capable of mediating cellular damage and their functional interaction mediates a cellular response capable of attenuating/mitigating the pathogenic potential of these cargos.

\section{Methods}

Patient samples and APOL1 genotyping. Archived paraffin-embedded human kidney biopsy samples with various histopathologic diagnosis (Supplemental Table 1) assigned by a clinical pathologist were obtained for immunohistology studies. For in situ hybridization and immunoelectron microscopy experiments, normal human kidney tissue adjacent to resected tumor tissue was collected as soon as possible after the operative procedure to reduce RNA degradation and ultrastructural changes. Age, gender, and self-reported race were obtained along with the tissue samples. Genotyping from paraffin-embedded biopsy specimens has been published previously (12). 
Reagents. Primary antibodies used for immunohistology and immunocytochemistry are as follows: rabbit polyclonal anti-APOL1 antibody (Sigma-Aldrich, catalog HPA018885, lot A39110, 1:50), goat polyclonal anti-VAMP8 antibody (R\&D Systems, catalog AF5354, lot CBVB01, 1:50), mouse monoclonal anti-GLEPP1 antibody (a gift from Roger Wiggins, University of Michigan, Ann Arbor, Michigan, USA), mouse monoclonal anti-ATP synthase subunit $\beta$ (Abcam, catalog ab14730, clone 3D5, 1:500), rabbit polyclonal anti-myc antibody (Upstate, catalog 06-549), and mouse monoclonal anti-LAMP1 antibody (R\&D Systems, catalog MAB4800, clone 508921, 1:50). Primary antibodies used for Western blot detection are as follows: rabbit polyclonal anti-APOL1 antibody (Sigma-Aldrich, catalog HPA018885, lot A39110), rabbit monoclonal antiAPOL1 antibody (Abcam, clone EPR2907[2]), rabbit polyclonal anti-myc antibody (Upstate, catalog 06-549), rabbit monoclonal anti-VAMP8 antibody (Abcam, clone EP2629Y), mouse monoclonal anti-FLAG antibody (Sigma-Aldrich, clone M2), and mouse monoclonal anti-GFP antibody (Clontech, clone JL8).

Immunohistochemistry, immunofluorescence, and immunocytochemistry. Immunohistology was performed as previously described (12); details are available in the Supplemental Methods. Briefly, human kidney tissue was formaldehyde fixed and paraffin embedded, and $5-\mu \mathrm{m}$ sections were collected on glass slides. Slides were deparaffinized and hydrated in alcohol gradients, followed by heat-induced antigen retrieval (HIAR) by heating in a pressure boiler in HIAR buffer $(10 \mathrm{mM}$ sodium citrate, $\mathrm{pH}=6.0,0.05 \%$ Tween-20). Slides were washed, and, if used for immunoperoxidase staining, endogenous peroxidase activity was quenched with 3\% hydrogen peroxide in PBS with $0.2 \%$ Tween-20. Slides were blocked and then incubated with primary antibody overnight and then secondary antibody for 1 hour. The Vectastain ABC kit (Vector Labs) was used according to the manufacturer's instructions for immunohistochemistry. For immunofluorescent microscopy, nuclei were labeled using TOTO-3-iodide (Invitrogen) and mounted with Vectashield (Vector Labs). Slides for immunofluorescent microscopy were viewed, and images were captured using a Leica TCS SP2 confocal system (Leica Microsystems). For immunohistochemistry, images were viewed using a Nikon Eclipse E600 microscope (Nikon Instruments Inc.), and images were captured with a SPOT RT3 camera using SPOT v5.1 software (SPOT Imaging Solutions). For immunocytochemistry, cells on coverslips were fixed with $4 \%$ paraformaldehyde at $4^{\circ} \mathrm{C}$ for 15 minutes followed by permeabilization with $0.2 \%$ Triton X-100. Incubation with primary antibodies was carried out at $4^{\circ} \mathrm{C}$ overnight, followed by incubation with appropriate secondary antibodies for 1 hour at room temperature and imaging as described above.

In situ hybridization. In situ hybridization was performed as previously described (36, 37). Sense and antisense riboprobes were synthesized with T7 RNA polymerase from linearized plasmid and digoxigenin labeled using the DIG RNA labeling kit (Roche Life Sciences). APOL1 riboprobes correspond to 1,095 base pairs of APOL1, including nucleotides 185-1,279 from NM_003661, which span the majority of the coding sequence. Hybridization and wash conditions have been previously described $(36,37)$ and additional details are available in the Supplemental Methods. The maximum stringency of washes after hybridization was controlled, with $2 \mathrm{X} \mathrm{SSC}$ at $55^{\circ} \mathrm{C}$ three times each for 10 minutes. After washing, anti-digoxigenin color development was carried out with NBT/BCIP reagent.

Immunogold electron microscopy. Human kidney tissue was fixed in $4 \%$ PFA for 4 hours at $4^{\circ} \mathrm{C}$, washed in PBS, and dehydrated in alcohol gradients. After 95\% alcohol, tissue was infiltrated with 1:1 ratio of $95 \%$ ethanol and LR white medium overnight followed by a 1:2 ratio for 12 hours and pure LR white medium overnight at $4^{\circ} \mathrm{C}$. Samples were polymerized at $40^{\circ} \mathrm{C}$ for $48-72$ hours. Ultrathin sections were cut with a diamond knife and mounted on nickel grids coated with Formvar. Grids were washed with PBS, blocked with $0.1 \%$ BSA in PBS for 30 minutes at room temperature, and incubated overnight at $4^{\circ} \mathrm{C}$ with rabbit polyclonal anti-APOL1 antibody (1:40; lot A39110, Sigma-Aldrich) in 1\% BSA/PBS rabbit IgG (1:40). For control experiments, tissue was incubated with rabbit IgG instead of anti-APOL1 antibody. Grids were washed and incubated with $0.1 \%$ BSA for 15 minutes and labeled with $10 \mathrm{~nm}$ gold-conjugated goat anti-rabbit IgG in $0.1 \%$ BSA/PBS for 45 minutes at room temperature. Fixation after labeling was performed with $1 \%$ glutaraldehyde and stained briefly with uranyl acetate and lead citrate. Grids were dried and examined with an electron microscope at 60kv (Philips CM12) at the Cleveland Clinic Lerner Research Electron Microscopy Core. Immunogold particles were counted and quantified as described in the Supplemental Methods.

Immunoprecipitation. Cells transfected with GFP-tagged APOL1 and myc-FLAG-tagged VAMP8 constructs were washed in PBS and lysed with IP/Pull-Down buffer $(10 \mathrm{mM}$ Tris-HCl, $\mathrm{pH}=7.5,200 \mathrm{mM}$ $\mathrm{NaCl}, 2 \mathrm{mM}$ EDTA, 0.5\% NP40, 0.01\% sodium deoxycholate, $1 \mathrm{mM}$ PMSF, and 1X protease inhibitor cocktail [Roche]), incubated at $4^{\circ} \mathrm{C}$ for $1-2$ hours, and centrifuged at $5,000 \mathrm{~g}$ for 10 minutes at $4^{\circ} \mathrm{C}$ and supernatant was collected and precleared with Gammabind Plus Sepharose beads for 3 hours. Immunopre- 
cipitation was done by incubating the precleared lysates with rabbit polyclonal anti-Myc antibody (Upstate, 1:20) or rabbit IgG as control overnight. Beads were washed eight times for 30 minutes at $4^{\circ} \mathrm{C}$ with wash buffer (5 mM Tris-HCl; $\mathrm{pH}=8,275 \mathrm{mM} \mathrm{NaCl}, 1 \mathrm{mM}$ EDTA, 0.05\% NP40) and then heated in Laemmli buffer at $85^{\circ} \mathrm{C}$ for 8 minutes, and proteins were resolved with SDS-PAGE.

Pull-down assay. Full-length APOL1-G0-V5-His, APOL1-G1-V5-His, and APOL1-G2-V5-His recombinant proteins for pulldown assays were expressed in bacterial cells using the pStaby system (Delphi Genetics; complete details provided in the Supplemental Methods). Pull-down experiments were performed using APOL1-G0, -G1, and -G2, which were all C-terminally tagged with V5-His and NHE1-His recombinant proteins bound to Talon beads. 293-T cells were transfected with VAMP8-Myc/FLAG construct. After 48 hours after transfection, cells were washed in PBS and lysed with IP/Pull-Down buffer, as described above. Lysate was incubated for 1 hour at $4^{\circ} \mathrm{C}$, and supernatant was collected after centrifuging at 5,000 $\mathrm{g}$ for 10 minutes at $4^{\circ} \mathrm{C}$. Supernatant was precleared with Talon beads for 3 hours at $4^{\circ} \mathrm{C}$, then incubated with recombinant APOL1 protein bound to beads for 4 hours at $4^{\circ} \mathrm{C}$ with constant agitation. Beads were washed eight times, 30 minutes each time, for 4 hours in $1.5 \mathrm{ml}$ of wash buffer $(5 \mathrm{mM}$ Tris- $\mathrm{HCl}, \mathrm{pH}=8,225 \mathrm{mM} \mathrm{NaCl}$, $1 \mathrm{mM}$ EDTA, and $0.05 \% \mathrm{NP} 40$ ) at $4^{\circ} \mathrm{C}$. Beads were heated in Laemmli buffer at $85^{\circ} \mathrm{C}$ for 8 minutes, and proteins were resolved using SDS-PAGE.

Western blots. Protein samples in Laemmli buffer were separated on Novex Tris-Glycine SDS-PAGE gel (Life Technologies) and transferred to a polyvinylidene difluoride membrane (Millipore). The membrane was blocked with 5\% nonfat dry milk (NFDM) in PBS with $0.2 \%$ Tween-20 and incubated with primary antibodies at $4{ }^{\circ} \mathrm{C}$ overnight in 5\% NFDM. After washing with $0.1 \%$ PBS-Tween-20, membrane was incubated with appropriate secondary antibody in 5\% NFDM for 1 hour at room temperature. Membrane was washed, and immunoreactive proteins were identified using Supersignal West Pico chemiluminescent substrate (Thermo Scientific). See complete unedited blots in the supplemental material.

Surface plasmon resonance. The interaction between APOL1 and VAMP8 was analyzed using the T100 BIAcore system (GE Healthcare Life Sciences). GST-APOL1 (aa 305-398) or GST alone (as control) was immobilized on a CM5 chip (GE Healthcare Life Sciences) by the amine coupling method. All flow cells (FC) were activated by injection of $50 \mathrm{mM} N$-hydroxysuccinimide and $200 \mathrm{mM} N$-ethyl- $N$ '-(3-diethylaminopropyl)-carbodimide for 7 minutes at a flow rate of $10 \mu \mathrm{l} / \mathrm{min}$. The following ligands were immobilized within each FC at 800 RU: GST (FC:1), GST-APOL1-G0 (aa 305-398) (FC:2), GST-APOL1-G1 (aa 305 398) (FC:3), and GST-APOL1-G2 (aa 305-398) (FC:4). After ligand immobilization, the surface was deactivated by injection of $1 \mathrm{M}$ ethanolamine at a flow rate of $10 \mu \mathrm{l} / \mathrm{min}$ for 7 minutes. Purified His-VAMP8 (aa 1-76) in $20 \mathrm{mM}$ HEPES, $200 \mathrm{mM} \mathrm{NaCl}, 2 \mathrm{mM}$ EDTA, $2 \mathrm{mM}$ TCEP, $0.02 \%$ P20 (pH = 7.4) was injected at a range of concentrations from FC: 1 to FC:4 at $30 \mu 1 /$ min for 2 minutes. The sensograms were analyzed using the BIAevaluation software, and equilibrium dissociation constants ( $\mathrm{Kd}$ values) were calculated from steady-state binding data after subtracting response from (FC:1), the control FC.

MD simulations. Initial structure of APOL1 C-terminus was obtained using the iterative threading assembly refinement server I-TASSER (Supplemental Table 3). Protein models were solvated in a TIP3P water box, and neutralizing ions were added. Following energy minimization, the system was equilibrated for 400 ps at $300 \mathrm{~K}$. Multiple simulations were performed using the CHARMM27 force field in NAMD v2.9, with a time step of $2 \mathrm{fs}$ (Supplemental Table 4). For nonbonded calculations, a cutoff distance of $12 \AA$ was used with a smooth switching function initiated at $10 \AA$. The standard particle-mesh Ewald method was applied to calculate the long-range electrostatic interactions. All simulations were carried out under isothermal-isobaric conditions (NPT ensemble) by maintaining temperature at $300 \mathrm{~K}$ by Langevin dynamics and constant pressure of $1 \mathrm{~atm}$ by employing the Nose-Hoover Langevin Piston method. Analysis of the MD simulation trajectories was carried out using VMD, Wordom, and Carma, and the results were plotted using Origin 9.1. An expanded discussion of the methods and experimental details is provided in the Supplemental Methods.

$C D$ spectroscopy. Recombinant proteins utilized in the $\mathrm{CD}$ experiments were generated in a bacterial expression system (complete details provided in the Supplemental Methods). The secondary structural content of the carboxy terminal portion (aa 305-398) of APOL1-G0, -G1, and -G2 proteins in CD buffer (20 mM potassium phosphate, $50 \mathrm{mM}$ sodium sulfate, $\mathrm{pH}=7.0$ ) was measured with far UV-CD spectra recorded at $25^{\circ} \mathrm{C}$ in a quartz cuvette with a $1-\mathrm{mm}$ path length. The secondary structure composition was estimated with Dichroweb using the CONTIN program (38-40). The APOL1 protein concentrations of samples used for CD experiments were measured by quantitative amino acid analysis (New England Peptides). Urea titration experiments were performed to determine stability of protein structure in the presence 
of denaturants. APOL1 protein samples were dialyzed overnight in CD buffer and then diluted with fresh $\mathrm{CD}$ buffer to a final protein concentration of $3 \mu \mathrm{M}$. The urea concentration in each sample was increased by stepwise increments of $0.1 \mathrm{M}$, increasing urea concentration from 0 to $8 \mathrm{M}$, while maintaining the protein concentration and $\mathrm{CD}$ buffer composition otherwise constant. $\mathrm{CD}$ signal at $222 \mathrm{~nm}$ was recorded at $37^{\circ} \mathrm{C}$ in a quartz cuvette with a $1-\mathrm{cm}$ path length at each of the $0.1 \mathrm{M}$ urea titration steps. Free energy of folding $\left(\Delta G_{0}\right)$ was calculated by linear extrapolation of free energy of the protein in the presence of $i$ th concentration of denaturant $\left(\Delta \mathrm{G}_{\mathrm{i}}\right)$ at the transition region as described previously $(41,42)$. All measurements were carried out in an Aviv spectrometer in the far UV absorption region (190-250 nm), with a 1-nm band width 1-mm slit width, using a cuvette of $10-\mathrm{mm}$ path length.

Cell stress assay. COS-7 cells (ATCC) were cultured to $40 \%-60 \%$ confluence on glass coverslips and cotransfected with expression plasmids for full-length $A P O L 1-G 0$ with a carboxy terminal GFP tag and fulllength $A P O L 1-G 2$ with a carboxy terminus $m$ Cherry tag. Cells were cultured for an additional 48 hours before fixing with $4 \%$ paraformaldehyde for 5 minutes at room temperature and mounting on slides for visualization of expressed GFP and mCherry. Cells were treated during the last 15 hours with rapamycin solubilized in ethanol at a final concentration of $0.2 \mu \mathrm{g} / \mathrm{ml}$ or during the last 6 hours with oligomycin $(0.5 \mu \mathrm{M})$, lipopolysaccharide $(1 \mathrm{ng} / \mathrm{ml})$, chloroquine $(20 \mu \mathrm{M})$, tunicamycin $(20 \mu \mathrm{g} / \mathrm{ml})$, and thapsigargin $(3 \mu \mathrm{M})(43,44)$.

Statistics. The statistical significance of difference in immunogold labeling between human kidney biopsy sections incubated with anti-APOL1 antibody or rabbit IgG (control) was analyzed by the Wilcoxon rank-sum test. Quantitative data are presented as a box plot in Supplemental Figure 6C. Statistical tests were performed using Microsoft Excel and SPSS. All reported $P$ values were 2 sided. $P$ values below 0.05 were considered statistically significant.

Study approval. All studies involving human tissues were performed with approval of the institutional review board of the MetroHealth System Campus, Case Western Reserve University.

\section{Author contributions}

SMM, JFO, LAB, MB, and JRS designed and conducted experiments, acquired and analyzed data, and prepared the manuscript. MK designed and conducted experiments, acquired and analyzed data, and reviewed manuscript. LB, DBT, and SG provided reagents, analyzed data, and reviewed manuscript.

\section{Acknowledgments}

This work was supported in part by National Institutes of Health grants DK097836 and DK108329 to LAB, JFO, and JRS and GM112491 to MB. SMM was supported by National Institutes of Health grant T32 DK007470. This work made use of the High Performance Computing Resource in the Core Facility for Advanced Research Computing at Case Western Reserve University.

Address correspondence to: John R. Sedor, 2500 MetroHealth Drive R415, Cleveland, Ohio 44109-1998, USA. Phone: 216.778.4993; Email: john.sedor@case.edu. Or to: Matthias Buck, 10900 Euclid Avenue E607, Cleveland, Ohio 44106, USA. Phone: 216.368.8651; Email: matthias.buck@case.edu.

1. Grams ME, Chow EK, Segev DL, Coresh J. Lifetime incidence of CKD stages 3-5 in the United States. Am J Kidney Dis. $2013 ; 62(2): 245-252$.

2. Genovese G, et al. Association of trypanolytic ApoL1 variants with kidney disease in African Americans. Science. 2010;329(5993):841-845.

3. Tzur S, et al. Missense mutations in the APOL1 gene are highly associated with end stage kidney disease risk previously attributed to the MYH9 gene. Hum Genet. 2010;128(3):345-350.

4. Parsa A, et al. APOL1 risk variants, race, and progression of chronic kidney disease. N Engl J Med. 2013;369(23):2183-2196

5. Kao WH, et al. MYH9 is associated with nondiabetic end-stage renal disease in African Americans. Nat Genet. 2008;40(10):1185-1192.

6. Iyengar SK, et al. Genome-wide association and trans-ethnic meta-analysis for advanced diabetic kidney disease: Family Investigation of Nephropathy and Diabetes (FIND). PLoS Genet. 2015;11(8):e1005352.

7. Schell JO, Da Silva-Gane M, Germain MJ. Recent insights into life expectancy with and without dialysis. Curr Opin Nephrol Hypertens. 2013;22(2):185-192.

8. Ruchi R, et al. Copy number variation at the APOL1 locus. PLoS One. 2015;10(5):e0125410.

9. Kopp JB, et al. APOL1 genetic variants in focal segmental glomerulosclerosis and HIV-associated nephropathy. $J$ Am Soc Nephrol. 2011;22(11):2129-2137.

10. Pérez-Morga D, et al. Apolipoprotein L-I promotes trypanosome lysis by forming pores in lysosomal membranes. Science. 2005;309(5733):469-472. 
11. Vanhamme L, et al. Apolipoprotein L-I is the trypanosome lytic factor of human serum. Nature. 2003;422(6927):83-87.

12. Madhavan SM, O'Toole JF, Konieczkowski M, Ganesan S, Bruggeman LA, Sedor JR. APOL1 localization in normal kidney and nondiabetic kidney disease. J Am Soc Nephrol. 2011;22(11):2119-2128.

13. Ma L, et al. Localization of APOL1 protein and mRNA in the human kidney: nondiseased tissue, primary cells, and immortalized cell lines. J Am Soc Nephrol. 2015;26(2):339-348.

14. Kotb AM, et al. Knockdown of ApoL1 in zebrafish larvae affects the glomerular filtration barrier and the expression of nephrin. PLoS One. 2016;11(5):e0153768.

15. Bruggeman LA, et al. Plasma apolipoprotein L1 levels do not correlate with CKD. J Am Soc Nephrol. 2014;25(3):634-644.

16. Kozlitina J, et al. Plasma levels of risk-variant APOL1 do not associate with renal disease in a population-based cohort. $J$ Am Soc Nephrol. 2016;27(10):3204-3219.

17. Freedman BI, et al. Apolipoprotein L1 gene variants in deceased organ donors are associated with renal allograft failure. Am $J$ Transplant. 2015;15(6):1615-1622.

18. Lee BT, et al. The APOL1 genotype of African American kidney transplant recipients does not impact 5-year allograft survival. Am J Transplant. 2012;12(7):1924-1928.

19. Pays E, Vanhollebeke B, Uzureau P, Lecordier L, Pérez-Morga D. The molecular arms race between African trypanosomes and humans. Nat Rev Microbiol. 2014;12(8):575-584.

20. Sharma AK, Friedman DJ, Pollak MR, Alper SL. Structural characterization of the C-terminal coiled-coil domains of wild-type and kidney disease-associated mutants of apolipoprotein L1. FEBS J. 2016;283(10):1846-1862.

21. Roy A, Kucukural A, Zhang Y. I-TASSER: a unified platform for automated protein structure and function prediction. Nat Protoc. 2010;5(4):725-738.

22. Freedman BI, et al. APOL1 genotype and kidney transplantation outcomes from deceased African American donors. Transplantation. 2016;100(1):194-202.

23. Bruggeman LA, et al. APOL1-G0 or APOL1-G2 transgenic models develop preeclampsia but not kidney disease. $J$ Am Soc Nephrol. 2016;27(12):3600-3610.

24. Beckerman P, et al. Transgenic expression of human APOL1 risk variants in podocytes induces kidney disease in mice. Nat Med. 2017;23(4):429-438.

25. Rossi V, et al. VAMP subfamilies identified by specific R-SNARE motifs. Biol Cell. 2004;96(4):251-256.

26. Limou S, Dummer PD, Nelson GW, Kopp JB, Winkler CA. APOL1 toxin, innate immunity, and kidney injury. Kidney Int. 2015;88(1):28-34

27. Vanwalleghem G, et al. Coupling of lysosomal and mitochondrial membrane permeabilization in trypanolysis by APOL1. Nat Commun. 2015;6:8078.

28. Lugli EB, Pouliot M, Portela Mdel P, Loomis MR, Raper J. Characterization of primate trypanosome lytic factors. Mol Biochem Parasitol. 2004;138(1):9-20.

29. Buck M, Xu W, Rosen MK. A two-state allosteric model for autoinhibition rationalizes WASP signal integration and targeting J Mol Biol. 2004;338(2):271-285.

30. Pays E, Vanhollebeke B, Vanhamme L, Paturiaux-Hanocq F, Nolan DP, Pérez-Morga D. The trypanolytic factor of human serum. Nat Rev Microbiol. 2006;4(6):477-486.

31. Stephens NA, Hajduk SL. Endosomal localization of the serum resistance-associated protein in African trypanosomes confers human infectivity. Eukaryotic Cell. 2011;10(8):1023-1033.

32. Molina-Portela Mdel P, Lugli EB, Recio-Pinto E, Raper J. Trypanosome lytic factor, a subclass of high-density lipoprotein, forms cation-selective pores in membranes. Mol Biochem Parasitol. 2005;144(2):218-226.

33. Pryor PR, et al. Combinatorial SNARE complexes with VAMP7 or VAMP8 define different late endocytic fusion events. EMBO Rep. 2004;5(6):590-595.

34. Kruzel-Davila E, et al. APOL1-mediated cell injury involves disruption of conserved trafficking processes. J Am Soc Nephrol. 2017;28(4):1117-1130

35. Fu Y, et al. APOL1-G1 in nephrocytes induces hypertrophy and accelerates cell death. J Am Soc Nephrol. 2017;28(4):1106-1116.

36. Ross MD, Martinka S, Mukherjee A, Sedor JR, Vinson C, Bruggeman LA. Math6 expression during kidney development and altered expression in a mouse model of glomerulosclerosis. Dev Dyn. 2006;235(11):3102-3109.

37. Bruggeman LA, et al. Renal epithelium is a previously unrecognized site of HIV-1 infection. J Am Soc Nephrol. 2000;11(11):2079-2087.

38. Whitmore L, Wallace BA. Protein secondary structure analyses from circular dichroism spectroscopy: methods and reference databases. Biopolymers. 2008;89(5):392-400.

39. Provencher SW, Glöckner J. Estimation of globular protein secondary structure from circular dichroism. Biochemistry. 1981;20(1):33-37.

40. van Stokkum IH, Spoelder HJ, Bloemendal M, van Grondelle R, Groen FC. Estimation of protein secondary structure and error analysis from circular dichroism spectra. Anal Biochem. 1990;191(1):110-118.

41. Padmanabhan S, et al. Thermodynamic analysis of the structural stability of phage 434 Cro protein. Biochemistry. 1999;38(47):15536-15547.

42. Greenfield NJ. Determination of the folding of proteins as a function of denaturants, osmolytes or ligands using circular dichroism. Nat Protoc. 2006;1(6):2733-2741.

43. Ganley IG, Wong PM, Gammoh N, Jiang X. Distinct autophagosomal-lysosomal fusion mechanism revealed by thapsigargin-induced autophagy arrest. Mol Cell. 2011;42(6):731-743.

44. Molle D, et al. Endosomal trafficking of HIV-1 gag and genomic RNAs regulates viral egress. J Biol Chem. 2009;284(29):19727-19743. 